\title{
Neuroplasticity-targeted Intervention for Idiopathic Sudden Sensorineural Hearing Loss: A New Therapeutic Direction
}

\author{
Chin-Lung Kuo, MD, PhD* \\ Institute of Brain Science, National Yang-Ming University, Taipei; Department of Otolaryngology, Taoyuan Armed Forces \\ General Hospital, Taoyuan, Taiwan
}

Abstract

Sudden sensorineural hearing loss (SSNHL) can be a frightening experience for patients, and practitioners have yet to arrive at a consensus with regard to the most appropriate course of treatment for this condition. This can be attributed primarily to a lack of understanding pertaining to the true etiopathogenesis of SSNHL. Given that the management of idiopathic SSNHL presents a challenge for clinicians and neuroscientists alike, research pertaining to SSNHL therapy may benefit from alternative approaches to this problem. This current review addresses the topic of therapeutic strategies for idiopathic SSNHL from the perspective of neuroplasticity. Assertions pertaining to the plausibility of this approach are based on a large body of evidence from animal experiments and recent studies on humans.

Keywords: acoustic; hearing loss; MEG; neuroplasticity; sound therapy

\section{Sudden Sensorineural Hearing Loss}

Sudden sensorineural hearing loss (SSNHL) is defined as a subjective sensation of rapid onset hearing impairment (i.e. occurring over a period less than 72 hours) that meets the following audiometric criteria: (1) a $\geq 30 \mathrm{~dB}$ decrease in hearing related to premorbid thresholds or (if premorbid audiometry is not available) opposite ear thresholds; and (2) at least 3 consecutive frequencies are affected [1].

SSNHL is an otologic condition first described in 1860 by Everberg, who reported a case of sudden deafness secondary to mumps [2]. SSNHL can be a frightening experience for patients, particularly those who depend on their hearing for their work, such as musicians, professional drivers, or athletes. Indeed, SSNHL can have a tremendous impact on one's quality of life, and has been correlated to an increased risk of adverse cognitive and functional outcomes. The number of new SSNHL cases is generally between 5 and 20 per 100,000 people per year; however, this figure is based on an 8-year prospective study on 225 patients, which was published in 1984 [3]. In 2004, a population-based cross-sectional study of SSNHL epidemiology in Germany reported that the incidence may be closer to 160 SSNHL cases per 100,000 people per year [4]. The higher number of SSNHL cases in the German study may be due to advances in screening and evaluation tools, which have enabled more definitive diagnoses of hearing-related diseases. Furthermore, the high incidence rate in the German study suggests that SSNHL is not a rare disease (as defined by the WHO and the European Union: less than 50 cases per 100,000 people) [4].

It has been estimated that, in Taiwan, there are approximately 2000 to 3000 new cases of SSNHL per year [5], whereas in the United States,

*Correspondence: Chin-Lung Kuo, MD, PhD

Institute of Brain Science, National Yang-Ming University, Taipei; Department of Otolaryngology, Taoyuan Armed Forces General Hospital, Taoyuan, Taiwan. Email: drkuochinlung@gmail.com

Received: March 8, 2017; Accepted: May 10, 2017; Published: May 20, 2017

Neurology and Neuroscience Research. 2017;1(1):1

DOI: 10.24983/scitemed.nnr.2017.00014

Copyright ( 2017 The Author(s). This is an open-access article distributed under the terms of the Creative Commons Attribution 4.0 International License (CC-BY). there are approximately 4000 new cases of SSNHL per year [6]. Nonetheless, it is likely that these numbers have been underestimated, given that many patients spontaneously recover without receiving medical attention [7]. All cases of spontaneous recovery occur within the first 2 weeks following the onset of symptoms $[1,6,8]$. Reports on the likelihood of spontaneous recovery vary considerably (between $32 \%$ and $65 \%$ ) $[3,6,9]$. Furthermore, spontaneous recovery has been shown to depend on several factors, including age, vestibular symptoms at onset, severity of hearing loss at presentation, and the amount of time that elapses between symptom onset and treatment [1]. SSNHL typically occurs in middle adulthood (43 to 53 years of age), and incidence increases with age $[3,5,7]$. Males and females are equally affected [7].

SSNHL can result from an abnormality of the cochlea, auditory nerve, or higher aspects of central auditory perception or processing [1], but the cochlea is thought to be most probable lesion site [10]. The underlying cause of SSNHL can only be identified in approximately $10 \%$ of cases; but in these cases, the most common causes have been found to be acoustic neuroma, stroke, and malignancy [11]. The remaining $90 \%$ of cases are idiopathic and presumptively attributed to vascular, infectious, immunologic, or multiple etiologies [1].

\section{Treatment Modalities for Idiopathic SSNHL}

The unclear etiology of SSNHL has led to the application of multiple therapy modalities, including systemic and intratympanic steroids, antiviral agents, anticoagulants, volume expanders, vasoactive substances, antioxidants, hyperbaric oxygen, anti-anxiety medication, diuretics (alone or in combination) [1], or observation alone [12]. Nonetheless, over the past three decades, a tapering course of corticosteroids (including prednisone, methylprednisolone, solumedrol, and dexamethasone) has been widely adopted as the principal treatment for idiopathic SSNHL [1]. The success rate of this treatment strategy is reported to be between 50 and $80 \%[7,13,14]$. However, recent systematic reviews of randomized controlled trials determined that the use of corticosteroids in SSNHL treatment is an issue of some controversy; i.e., these findings are based on conflicting results from multiple studies [1,9,15-17], and other treatment options have not been validated by sufficiently rigorous randomized trials [1].

The primary challenges related to the management and treatment of SSNHL can be attributed to insufficient understanding of its etiopathoge- 
nesis. Indeed, in the absence of an underlying cause that is known and treatable, the management of idiopathic SSNHL presents a challenge to clinicians and neuroscientists alike. The fact is that (1) SSNHL has serious consequences and negatively impacts one's quality of life $[1,18]$, and (2) no medication has proven effective in treating SSNHL [1]. Clearly, research into SSNHL therapy could benefit from new perspectives. This current review approaches the topic of therapeutic strategies for idiopathic SSNHL from the novel perspective of neuroplasticity. The biological plausibility of plastic therapeutic assumptions are based on a large body of evidence from animal experiments [19-23] and recent human studies [24,25].

\section{Neuroplasticity}

The word "plasticity" is derived from the Greek word "plastos", meaning molded [26]. Neuroplasticity or neural plasticity refers to the ability of the nervous system to reorganize its structure, function, and connections in response to environmental stimuli or demands [27]. The term "plasticity" was first introduced to the neurosciences in 1890 by American psychologist William James [28], who reported that nervous tissue appears to have an extraordinary degree of plasticity, and that, in living beings, the phenomenon of habit is due to plasticity $[26,28]$. When the concept of brain plasticity was first introduced, it challenged the belief that the structure of the brain is relatively unchangeable after a critical period during early childhood, which was the dominant belief among neuroscientists of the day. In 1904, Santiago Ramón y Cajal, a Spanish Nobel prize winner and the father of modern neuroscience published a book entitled "Textura del Sistema Nervioso", extended the notion of plasticity to the neural substrate by reporting that, when an individual acquires new skills, the brain changes by reinforcing preexisting connections and then forms new pathways [26]. By the middle of the $20^{\text {th }}$ century, efforts to prove or disprove the concept of neuroplasticity led to innovative breakthroughs in experimental design, which became the cornerstones of neuroplasticity theory. In 1949, Canadian psychologist and father of neuropsychology Donald Olding Hebb proposed Hebbian theory, which posits that the brain exhibits synaptic plasticity during the learning process [29]. He made the following claims: "When an axon of cell $A$ is near enough to excite a cell $B$ and repeatedly or persistently takes part in firing it, some growth process or metabolic change takes place in one or both cells such that A's efficiency, as one of the cells firing $B$, is increased." The Hebbian activity-dependent refinement model is commonly referred to as Hebb's Law and can be summarized as: "Cells that fire together, wire together." In brief, Hebb's Law posits that excitatory connections are formed between coactive presynaptic and postsynaptic cells. The Hebbian prediction that coactive inputs are stabilized was supported by evidence from a recent study by Munz, et al., who presented live observations of axonal structural plasticity directed by patterned visual stimuli in vivo [30]. Hebbian theory presented a significant early challenge to the conventional wisdom of fixed-brain theory. However, it was not until the late 1960s that Raisman introduced the term "neuronal plasticity" to describe a permanent plastic change that he observed in the neuropils of septal nuclei of adult rats in response to deafferentation. Decades of research has shown that the brain responds to various internal and external stimuli dynamically [31]. Moreover, recent studies have indicated that although neuroplasticity underlies a fundamental lifelong property of the nervous system, it is particularly common during the developmental stage and underlies the ability of children to learn quickly [26,27].

Neuroplasticity involves adaptive responses to conditions resulting in a behavioral gain, such as learning. Gaser and Schlaug used a voxel-byvoxel morphometric technique to compare professional musicians (keyboard players) with a matched group of amateur musicians and nonmusicians [32]. They found that professional musicians had the largest volume of gray matter in the motor, auditory, and visual-spatial regions of the brain involved in playing music, followed by amateur musicians and non-musicians. A study by Woollett, et al. [33] revealed that the hippocampus, which plays a role in navigating large-scale spatial environments [34], of taxi drivers in London is larger than that of bus drivers. This finding may be explained by the fact that taxi drivers must navigate busy London traffic and typically use a different route every time they deal with a new customer; while bus drivers operate along a constrained set of routes. The authors concluded that neuropsychological and structural brain changes can occur after extensive mental effort. Finally, Pantev, et al. used functional magnetic source imaging (single dipole model) to measure cortical representations in highly skilled musicians. They found that training-induced functional reorganization extended farther across the sensory cortices of musicians than across that of control subjects who had never played an instrument. Findings from that study revealed that tonotopic representations can undergo dynamic changes, particularly after the training of skills [35]. Several other studies on humans and animals have also reported strong links between use-dependent structural adaptations in the brain and extensive training efforts [36-40].

\section{Plasticity-promoting Interventions}

Plastic change is an adaptive gain in function associated with the performance of a skill or the retention of a memory. It may occur during healthy development or during recovery from brain damage. For example, following a brain lesion, cortical reorganization may increase residual function or otherwise compensate for the loss of function, such that initial deficits in behavior, perceptual, and/or cognitive skills present signs of improvement over time [41]. The recovering, re-normalizing entity of neuroplasticity offers a promising intervention strategy for clinical applications. Specifically, neuroplasticity can be harnessed to achieve therapeutic gains by reversing maladaptive cortical reorganization [25-27,42-44]. Given that plasticity-promoting theory has been implicated in the alleviation of clinical disorders, several promising neuroplasticity-based interventions aimed at enhancing brain plasticity have been proposed. These include the principles of Hebbian learning [45], task-specific training [46], transcranial magnetic stimulation [47], deep brain stimulation [48], cognitive behavioral therapy [49], physical training [50], and neuropharmacotherapies that involve the molecular manipulation of cellular and synaptic pathways $[27,51,52]$. These interventions have been shown to promote clinical gains, improve behavioral outcomes, and increase brain plasticity. However, the extent of behavioral benefits conferred by plasticity-promoting interventions depends on the availability of sufficient residual neural capacity, rather than the type or duration of neurological insult $[27,53,54]$.

\section{Plasticity-preventing Interventions}

Advances in the field of brain plasticity have led to the development of promising interventions for severe neurological conditions and disorders. These interventions promote adaptive neuroplastic changes to compensate for lost functions or to maximize remaining functions. Nonetheless, our understanding of the complexity and multidimensionality of neuroplasticity is incomplete. Some adaptive plastic changes are associated with behavioral gain (e.g. skill learning) or functional compensation (e.g. post-stroke recovery), whereas other forms of plasticity can induce maladaptive neuroplastic changes and negatively affect disease pathogenesis $[25,27,41,55-57]$. Notable examples of maladaptive cortical reorganization include focal hand dystonia [58], phantom limb pain [59], and tinnitus $[60,61]$, which can have disastrous effects on one's quality of life [41]. Most strategies addressing the adverse consequences of plasticity involve prevention rather than promotion. Behavioral training has been shown to reduce or to reverse maladaptive cortical reorganization. Collectively, maladaptive plastic changes can have a strong influence over undesirable patterns of cortical activation [26]. Interventions aimed at moderating the effects of plasticity can also help to elucidate maladaptive cortical reorganization.

\section{Neuroplasticity-targeted Interventions for Idiopathic SSNHL}

As noted above, neuroplasticity can have positive as well as negative effects $[41,61]$. Nonetheless, the gain in functional recovery can be improved through the promotion of adaptive cortical reorganization or through the prevention of maladaptive plastic changes. In this regard, neuroplasticity-based interventions represent a promising therapeutic direction for the management of neurological disorders.

No medications have been proven successful in the treatment of SSNHL. Even steroids (the most commonly "standard" treatment option), lack evidence-based proof of efficacy [1]. Furthermore, many patients do not qualify as candidates for steroids due to the potentially severe side effects associated with this treatment, including the suppression of hypothalamic-pituitary-adrenal function, insomnia, weight gain, gastritis, 
mood changes, hyperglycemia, hypertension, cataracts, opportunistic infections, osteoporosis, and osteonecrosis [1,62].

SSNHL can be a frightening incident causing embarrassment, frustration, anxiety, insecurity, loneliness, depression, and social isolation [1,63]. Treatment limitations and the potentially serious consequences of SSNHL highlight the need to explore new therapeutic strategies. Neuroplasticitybased interventions may represent a feasible approach.

\section{Neuroplasticity-targeted Interventions in Animal Models}

Neuropathogenic mechanisms underlying SSNHL were used in the development of a revolutionary approach to treatment based on neuroplasticity. In animal models, acoustic trauma has been shown to induce hearing loss via damage to the cochlea $[64,65]$ and auditory nerve fibers $[66,67]$. The resulting decrease in the rates of firing (spontaneous and driven) in auditory nerve fibers can lead to central reorganization $[22,23,68]$. Researchers have also revealed that animals subjected to auditory stimulation following acoustic trauma are less affected by hearing loss and hair cell damage in the cochlea, compared to animals that are not subjected to post-traumatic acoustic stimulation $[19,20,22]$. One recent study reported that cats exposed to traumatizing noise presented changes in tonotopic organization in the primary auditory cortex, whereas the cortical tonotopic map of cats in a quiet environment presented no signs of reorganization [22]. These studies strongly indicate that changes in brain plasticity may be triggered by a decline in the spontaneous and driven firing rates in auditory nerve fibers following cochlear damage [22]. Using neuroplasticity-targeted interventions, therefore, acoustic energy delivered to ciliated cells in the cochlea is converted into electrical impulses that are transmitted to the auditory cortex through auditory nerves [24]. The form of rehabilitating mechanism of neuroplasticity-targeted interventions may (1) compensate for the loss of afferent neural inputs induced by deafferentation and (2) prevent maladaptive neuroplasticity, thereby enhancing the process of hearing recovery and facilitating hearing gain [24,25].

\section{Neuroplasticity-targeted Interventions in Human Models}

In recent functional magnetic resonance imaging (fMRI) studies on humans $[69,70]$, using acoustic stimulation was shown to alter the auditory cortical response of patients suffering from sudden unilateral deafness. Immediate and protracted changes in auditory pathway functions have also been observed in studies on magnetoencephalography (MEG) [71-75]. These studies indicate that humans can undergo similar SSNHL-induced brain plasticity as that observed in animal studies. $\mathrm{Li}$, et al. further observed altered hemispheric plastic asymmetry with a pattern of "healthy-side dominance" of N100 response (N100m in MEG) to tone burst stimulation in patients with early stage idiopathic SSNHL. Conversely, subjects with normal hearing showed "contralateral dominance" $[10,75]$. These initial observations of MEG hemispheric asymmetry can be interpreted as evidence of active compensation to facilitate the recovery of hearing.

In 2012, López-González, et al. published a study comparing the outcomes of $65 \mathrm{SSNHL}$ patients treated with medication only (steroids, piracetam, or antioxidants) and $67 \mathrm{SSNHL}$ patients treated with medication in conjunction with sound therapy (a combination of music and speech) [24]. Sound therapy was shown to greatly enhance the effectiveness of treatment on idiopathic SSNHL patients. Unfortunately, they analyzed audiometric outcomes without using neuroimaging methods, such as $\mathrm{fMRI}$ or MEG, thereby precluding the possibility of determining the extent of changes due to brain plasticity.

In 2014, Okamoto, et al. designed a modified form of sound therapy, referred to as "constraint-induced sound therapy (CIST)," for patients with idiopathic SSNHL [25]. CIST was designed in accordance with a wellestablished approach to neuro-rehabilitation, known as "constraintinduced movement therapy." This method has been shown to improve chronic motor deficit in patients with stroke. CIST includes two major components: (1) plugging the intact ear to ensure that the cochlea does not receive acoustic (mechanical) inputs, thereby preventing the transmission of afferent electrical neural impulses to the contralateral auditory cortex; (2) stimulating the affected ear with music (six hours per day) to prevent maladaptive auditory cortical plasticity on the healthy side. They also compared pure tone audiograms obtained from patients who underwent constraint-induced sound therapy combined with standard corticosteroid therapy $(C I S T+S C T, n=22)$ with those of patients who underwent standard corticosteroid therapy only (SCT, n=31). In that study, the hearing recovery of the CIST+SCT group far exceeded that of the SCT group. They also used MEG to analyze the neural activity of six patients from the CIST+SCT group, focusing on the following types of neural activity: $\mathrm{N} 1 \mathrm{~m}$ response (generated mainly in the belt and parabelt areas of the auditory cortex) [76] and the auditory steady state response (generated in the primary auditory cortex) [77]. They found that monaural stimulation induced contralateral dominance, which is indicative of normal hearing. This suggests that treatment with CIST+SCT reversed contralateral cortical maladaptive plastic changes induced by SSNHL $[10,75]$. Clearly, acoustic stimulation could play a significant role in neuroplasticity-targeted interventions for idiopathic SSNHL by preventing as well as reversing the adverse effects of maladaptive cortical plasticity. Nonetheless, the study by Okamoto, et al. suffered from important limitations. To begin with, only 6 of the $53(11 \%)$ patients chose to undergo an MEG test, which may have resulted in a biased sample. These findings should therefore be validated in future research with a larger patient population. Second, audiometric data for the six patients who underwent MEG were not available. Thus, it may be premature to conclude that there is an association between hearing recovery and reversion of cortical reorganization.

The only studies on SSNHL interventions with acoustic stimulation were those of López-González, et al. [24] and Okamoto, et al. [25]. Thus far, all attempts to validate the postulated theory (i.e., that sound therapy may prevent or reverse SSNHL-induced maladaptive cortical reorganization) have failed [25]. Furthermore, all of the patients in these studies also underwent steroid treatment (which is the current first-line treatment); therefore, functional recovery cannot be attributed solely to sound therapy. At present, steroid therapy has a success rate of approximately $50 \%$ to $80 \%$ [14]. Furthermore, the large number of patients that undergo spontaneous recovery ( $32 \%$ to $65 \%$ ) makes it very difficult to determine the degree to which acoustic stimulation actually improves hearing function $[3,6,9]$.

\section{Future Work}

Evidence from animal models suggests that acoustic stimulation may interrupt the mechanisms that contribute to central reorganization, thereby helping to protect the auditory system from further damage. Indeed, the success achieved in animal models has encouraged scientists to employ similar strategies in the study of human with SSNHL. Although sound therapy has been applied on human subjects and we now have a far better understanding of the neuroplasticity-targeted interventions for idiopathic SSNHL, the theoretical hypothesis that sound therapy can improve SSNHL outcomes has not been tested in a sufficiently rigorous manner. Further evidence confirming the therapeutic effects of sound therapy on hearing protection is needed.

In the future, we hypothesize that hearing loss in patients with idiopathic SSNHL could be restored by boosting audio levels targeting specific frequencies while constraining the transmission of signals (at corresponding frequencies) to the unaffected ear. Evidence obtained in animal studies indicates that the mechanism underlying hearing restoration may involve the model of neuroplasticity-prevention $[19,20,22]$.

\section{Conclusions}

This review introduced acoustic models aimed at elucidating the mechanisms underlying the process of hearing restoration induced by acoustic stimulation. The hypothesis of neuroplasticity-prevention states that hearing restoration may be in attempt to provide neurophysiological implications of neuroprotective acoustic training in patients with idiopathic SSNHL.

\section{Article Information}

Conflict of Interest Disclosures: The author has no conflicts of interest to disclose.

Funding: This study was sponsored by grant 10626 from Taoyuan Armed Forces General Hospital. 


\section{References}

1. Stachler RJ, Chandrasekhar SS, Archer SM, et al. Clinical practice guide-line: sudden hearing loss. Otolaryngology--head and neck surgery: official journal of American Academy of OtolaryngologyHead and Neck Surgery 2012;146:S1-35.

2. Nogueira JF, Jr., Hermann DR, Americo Rdos R, Barauna Filho IS, Stamm AE, Pignatari SS. A brief history of otorhinolaryngolgy: otology, laryngology and rhinology. Brazilian journal of otorhinolaryngology 2007;73:693-703.

3. Byl FM, Jr. Sudden hearing loss: eight years' experience and suggested prognostic table. The Laryngoscope 1984;94:647-661.

4. Klemm E, Deutscher A, Mosges R. [A present investigation of the epidemiology in idiopathic sudden sensorineural hearing loss]. Laryngo- rhino- otologie 2009;88:524-527.

5. Wu CS, Lin HC, Chao PZ. Sudden sensorineural hearing loss: evidence from Taiwan. Audiology \& neuro-otology 2006;11:151-156.

6. Mattox DE, Simmons FB. Natural history of sudden sensorineural hearing loss. The Annals of otology, rhinology, and laryngology 1977;86:463-480.

7. Rauch SD, Halpin CF, Antonelli PJ, et al. Oral vs intratympanic corticosteroid therapy for idiopathic sudden sensorineural hearing loss: a randomized trial. Jama 2011;305:2071-2079.

8. Rauch SD. Clinical practice. Idiopathic sudden sensorineural hearing loss. The New England journal of medicine 2008;359:833-840.

9. Conlin AE, Parnes LS. Treatment of sudden sensorineural hearing loss: II. A Meta-analysis. Archives of otolaryngology--head \& neck surgery 2007;133:582-586.

10. Li LP, Shiao AS, Chen LF, et al. Healthy-side dominance of middle- and long-latency neuromagnetic fields in idiopathic sudden sensorineural hearing loss. The European journal of neuroscience 2006;24:937-946.

11. Saunders JE, Luxford WM, Devgan KK, Fetterman BL. Sudden hearing loss in acoustic neuroma patients. Otolaryngology--head and neck surgery: official journal of American Academy of OtolaryngologyHead and Neck Surgery 1995;113:23-31.

12. Guyot JP, Thielen K. [Evolution of sudden deafness without treatment] Schweizerische medizinische Wochenschrift. Supplementum 2000;116:93S-96S.

13. Wilson WR, Byl FM, Laird N. The efficacy of steroids in the treatment of idiopathic sudden hearing loss. A double-blind clinical study. Archives of otolaryngology 1980;106:772-776.

14. Ferri E, Frisina A, Fasson AC, Armato E, Spinato G, Amadori M. Intratympanic steroid treatment for idiopathic sudden sensorineural hearing loss after failure of intravenous therapy. ISRN otolaryngology 2012:2012:647271.

15. Wei BP, Stathopoulos D, O'Leary S. Steroids for idiopathic sudden sensorineural hearing loss. The Cochrane database of systematic reviews 2013;7:CD003998.

16. Conlin AE, Parnes LS. Treatment of sudden sensorineural hearing loss: I. A systematic review. Archives of otolaryngology--head \& neck surgery 2007;133:573-581.

17. Labus J, Breil J, Stutzer $\mathrm{H}$, Michel O. Meta-analysis for the effect of medical therapy vs. placebo on recovery of idiopathic sudden hearing loss. The Laryngoscope 2010;120:1863-1871.

18. Chia EM, Wang J, Rochtchina E, Cumming RR, Newall P, Mitchell P. Hearing impairment and health-related quality of life: the Blue Mountains Hearing Study. Ear and hearing 2007;28:187-195.

19. Fukushima N, White P, Harrison RV. Influence of acoustic deprivation on recovery of hair cells after acoustic trauma. Hearing research 1990;50:107-118.

20. Niu X, Tahera Y, Canlon B. Protection against acoustic trauma by forward and backward sound conditioning. Audiology \& neuro-otology 2004;9:265-273.

21. Willott JF, Bross L. Effects of prolonged exposure to an augmented acoustic environment on the auditory system of middle-aged C57BL/6J mice: cochlear and central histology and sex differences. The Journal of comparative neurology 2004;472:358-370.

22. Norena AJ, Eggermont JJ. Enriched acoustic environment after noise trauma reduces hearing loss and prevents cortical map reorganization. The Journal of neuroscience : the official journal of the Society for Neuroscience 2005;25:699-705.
23. Rajan R, Irvine DR, Wise LZ, Heil P. Effect of unilateral partial cochlear lesions in adult cats on the representation of lesioned and unlesioned cochleas in primary auditory cortex. The Journal of comparative neurology 1993;338:17-49.

24. Lopez-Gonzalez MA, Cambil E, Abrante A, Lopez-Fernandez R, Esteban F. [Sound therapy in sudden deafness]. Acta otorrinolaringologica espanola 2012;63:165-172.

25. Okamoto $H$, Fukushima $M$, Teismann $H$, et al. Constraint-induced sound therapy for sudden sensorineural hearing loss--behavioral and neurophysiological outcomes. Scientific reports 2014;4:3927.

26. Pascual-Leone A, Amedi A, Fregni F, Merabet LB. The plastic human brain cortex. Annual review of neuroscience 2005;28:377-401.

27. Cramer SC, Sur M, Dobkin BH, et al. Harnessing neuroplasticity for clinical applications. Brain : a journal of neurology 2011;134:15911609.

28. William James. The Principles of Psychology. New York: Henry Holt, 1890.

29. Donald Olding Hebb. The Organization of Behavior. New York: Wiley \& Sons, 1949

30. Munz M, Gobert D, Schohl A, et al. Rapid Hebbian axonal remodeling mediated by visual stimulation. Science 2014;344:904-909.

31. Fuchs E, Flugge G. Adult neuroplasticity: more than 40 years of research. Neural plasticity 2014;2014:541870.

32. Gaser C, Schlaug G. Brain structures differ between musicians and non-musicians. The Journal of neuroscience : the official journal of the Society for Neuroscience 2003;23:9240-9245.

33. Woollett K, Spiers HJ, Maguire EA. Talent in the taxi: a model system for exploring expertise. Philosophical transactions of the Royal Society of London. Series B, Biological sciences 2009;364:1407-1416.

34. Bird CM, Burgess N. The hippocampus and memory: insights from spatial processing. Nature reviews. Neuroscience 2008;9:182-194.

35. Pantev C, Oostenveld R, Engelien A, Ross B, Roberts LE, Hoke M. Increased auditory cortical representation in musicians. Nature 1998;392:811-814.

36. Schneider P, Scherg M, Dosch HG, Specht HJ, Gutschalk A, Rupp A. Morphology of Heschl's gyrus reflects enhanced activation in the auditory cortex of musicians. Nature neuroscience 2002;5:688-694.

37. Pantev C, Roberts LE, Schulz M, Engelien A, Ross B. Timbre-specific enhancement of auditory cortical representations in musicians. Neuroreport 2001;12:169-174.

38. Mechelli A, Crinion JT, Noppeney U, et al. Neurolinguistics: structural plasticity in the bilingual brain. Nature 2004;431:757.

39. Anderson BJ, Eckburg PB, Relucio KI. Alterations in the thickness of motor cortical subregions after motor-skill learning and exercise. Learning \& memory 2002;9:1-9.

40. Kleim JA, Lussnig E, Schwarz ER, Comery TA, Greenough WT. Synaptogenesis and Fos expression in the motor cortex of the adult rat after motor skill learning. The Journal of neuroscience : the official journal of the Society for Neuroscience 1996;16:4529-4535.

41. Elbert T, Heim S. A light and a dark side. Nature 2001;411:139.

42. Rosenzweig ES, Courtine G, Jindrich DL, et al. Extensive spontaneous plasticity of corticospinal projections after primate spinal cord injury. Nature neuroscience 2010;13:1505-1510.

43. Cramer SC, Lastra L, Lacourse MG, Cohen MJ. Brain motor system function after chronic, complete spinal cord injury. Brain : a journal of neurology 2005;128:2941-2950.

44. Sullivan EV, Pfefferbaum A. Neurocircuitry in alcoholism: a substrate of disruption and repair. Psychopharmacology 2005;180:583-594.

45. Pennartz CM. Reinforcement learning by Hebbian synapses with adaptive thresholds. Neuroscience 1997;81:303-319.

46. Hubbard IJ, Parsons MW, Neilson C, Carey LM. Task-specific training: evidence for and translation to clinical practice. Occupational therapy international 2009;16:175-189.

47. Mantovani A, Aly M, Dagan Y, Allart A, Lisanby SH. Randomized sham controlled trial of repetitive transcranial magnetic stimulation to the dorsolateral prefrontal cortex for the treatment of panic disorder with comorbid major depression. Journal of affective disorders 2013;144:153-159.

48. Pepper J, Hariz M, Zrinzo L. Deep brain stimulation versus anterior 
capsulotomy for obsessive-compulsive disorder: a review of the literature. Journal of neurosurgery 2015;122(5):1028-1037.

49. Walkup JT, Albano AM, Piacentini J, et al. Cognitive behavioral therapy, sertraline, or a combination in childhood anxiety. The New England journal of medicine 2008;359:2753-2766.

50. Lo AC, Guarino PD, Richards LG, et al. Robot-assisted therapy for longterm upper-limb impairment after stroke. The New England journal of medicine 2010;362:1772-1783.

51. Vecsey CG, Hawk JD, Lattal KM, et al. Histone deacetylase inhibitors enhance memory and synaptic plasticity via CREB:CBP-dependent transcriptional activation. The Journal of neuroscience: the official journal of the Society for Neuroscience 2007;27:6128-6140.

52. Potter WB, O'Riordan $\mathrm{KJ}$, Barnett $\mathrm{D}$, et al. Metabolic regulation of neuronal plasticity by the energy sensor AMPK. PloS one 2010;5:e8996.

53. Cramer SC, Parrish TB, Levy RM, et al. Predicting functional gains in a stroke trial. Stroke; a journal of cerebral circulation 2007;38:21082114.

54. Riley JD, Le V, Der-Yeghiaian L, et al. Anatomy of stroke injury predicts gains from therapy. Stroke; a journal of cerebral circulation 2011;42:421-426.

55. Nudo RJ. Plasticity. NeuroRx : the journal of the American Society for Experimental NeuroTherapeutics 2006;3:420-427.

56. Bara-Jimenez W, Catalan MJ, Hallett M, Gerloff C. Abnormal somatosensory homunculus in dystonia of the hand. Annals of neurology 1998;44:828-831.

57. Merzenich MM, Van Vleet TM, Nahum M. Brain plasticity-based therapeutics. Frontiers in human neuroscience 2014;8:385.

58. Candia V, Elbert T, Altenmuller E, Rau H, Schafer T, Taub E. Constraintinduced movement therapy for focal hand dystonia in musicians. Lancet 1999;353:42.

59. Weiss T, Miltner WH, Adler T, Bruckner L, Taub E. Decrease in phantom limb pain associated with prosthesis-induced increased use of an amputation stump in humans. Neuroscience letters 1999;272:131134.

60. Okamoto H, Stracke H, Stoll W, Pantev C. Listening to tailor-made notched music reduces tinnitus loudness and tinnitus-related auditory cortex activity. Proceedings of the National Academy of Sciences of the United States of America 2010;107:1207-1210.

61. Pantev C, Okamoto H, Teismann H. Tinnitus: the dark side of the auditory cortex plasticity. Annals of the New York Academy of Sciences 2012;1252:253-258.

62. Liu $\mathrm{D}$, Ahmet $\mathrm{A}$, Ward $\mathrm{L}$, et al. A practical guide to the monitoring and management of the complications of systemic corticosteroid therapy. Allergy, asthma, and clinical immunology : official journal of the Canadian Society of Allergy and Clinical Immunology 2013;9:30.

63. Mosges R, Koberlein J, Erdtracht B, Klingel R, Group R-IS. Quality of life in patients with idiopathic sudden hearing loss: comparison of diffe- rent therapies using the Medical Outcome Short Form (36) Health Survey questionnaire. Otology \& neurotology : official publication of the American Otological Society, American Neurotology Society [and] European Academy of Otology and Neurotology 2008;29:769-775.

64. Liberman MC, Dodds LW. Acute ultrastructural changes in acoustic trauma: serial-section reconstruction of stereocilia and cuticular plates. Hearing research 1987;26:45-64.

65. Liberman MC. Chronic ultrastructural changes in acoustic trauma: serial-section reconstruction of stereocilia and cuticular plates. Hearing research 1987;26:65-88.

66. Puel JL, Ruel J, Gervais d'Aldin C, Pujol R. Excitotoxicity and repair of co-chlear synapses after noise-trauma induced hearing loss. Neuroreport 1998;9:2109-2114.

67. Pujol R, Puel JL. Excitotoxicity, synaptic repair, and functional recovery in the mammalian cochlea: a review of recent findings. Annals of the New York Academy of Sciences 1999;884:249-254.

68. Norena AJ, Eggermont JJ. Changes in spontaneous neural activity immediately after an acoustic trauma: implications for neural correlates of tinnitus. Hearing research 2003;183:137-153.

69. Suzuki M, Kouzaki H, Nishida Y, Shiino A, Ito R, Kitano H. Cortical representation of hearing restoration in patients with sudden deafness. Neuroreport 2002;13:1829-1832.

70. Bilecen D, Seifritz E, Radu EW, et al. Cortical reorganization after acute unilateral hearing loss traced by fMRI. Neurology 2000;54:765-767.

71. Vasama JP, Makela JP, Pyykko I, Hari R. Abrupt unilateral deafness modifies function of human auditory pathways. Neuroreport 1995;6:961-964.

72. Vasama JP, Makela JP. Auditory pathway plasticity in adult humans after unilateral idiopathic sudden sensorineural hearing loss. Hearing research 1995;87:132-140.

73. Fujiki $N$, Naito $Y$, Nagamine $T$, et al. Influence of unilateral deafness on auditory evoked magnetic field. Neuroreport 1998;9:3129-3133.

74. Dietrich V, Nieschalk M, Stoll W, Rajan R, Pantev C. Cortical reorganization in patients with high frequency cochlear hearing loss. Hearing research 2001;158:95-101.

75. Po-Hung Li L, Shiao AS, Lin YY, et al. Healthy-side dominance of cortical neuromagnetic responses in sudden hearing loss. Annals of neurology 2003;53:810-815.

76. Salminen $\mathrm{NH}$, Tiitinen $\mathrm{H}$, Miettinen I, Alku P, May PJ. Asymmetrical representation of auditory space in human cortex. Brain research 2010;1306:93-99.

77. Pantev C, Roberts LE, Elbert T, Ross B, Wienbruch C. Tonotopic organization of the sources of human auditory steady-state responses. Hearing research 1996;101:62-74. 